\title{
DOA estimation based on data level Multistage Nested Wiener Filter
}

\author{
Xiaodong $\mathrm{He}^{*}$, Jun Zhu and Bin Tang
}

\begin{abstract}
A novel direction of arrival (DOA) estimation method based on data level Multistage Nested Wiener Filters (MSNWF) which is used to adaptive beamforming for subarray signal is proposed in this paper. The subarrays using the same array geometry are used to form a signal whose phase relative to the reference signal is a function of the DOA. The DOA is estimated by computing the phase shift between the reference signal and its phase-shifted version. The performance of this DOA estimation method is significantly improved due to the application of MSNWF for rejecting interference signals. The computation of the proposed method is simple, and the number of detectable signal sources can exceed the number of antenna elements.
\end{abstract}

Keywords: DOA; Estimation; MSNWF; Beamforming; Subarrays

\section{Introduction}

The smart antenna has been widely used in many applications such as radar, sonar, and wireless communication systems in the last two decades [1-4]. In these sensor networks implication systems and scenarios, direction of arrival (DOA) is an important parameter needed to be the estimates to determine the direction of the located and tracked target or the position of the sensor nodes. Considerable research efforts have been made in the DOA estimation, and various array signal process techniques for DOA estimation have been proposed [5-9].

The traditional DOA estimation techniques meanly include: (1) spectrum-based methods, such as Bartlett [4] and Capon [5]; (2) subspace-based algorithm, such as multiple signal classification (MUSIC) [7]; and (3) parametric methods, such as estimation of signal parameters via rotational invariance technique (ESPRIT) [8-10]. In Capon techniques, the DOAs are determined by finding the directions in which their antenna response vectors lead to peaks in the spectrum formed by the covariance matrix of the observation vectors. However, the capacity of this DOA estimation technique is less than the number of antenna elements, which is bounded by the covariance matrix of the observation vectors. In MUSIC techniques, the DOAs of target signals are determined

\footnotetext{
* Correspondence: winter_he@hotmail.com

School of Electronic Engineering, University of Electronic Science and Technology of China, Chengdu 611731, China
}

by finding the directions in which their antenna response vectors lead to peaks in the MUSIC spectrum formed by the eigenvectors of the noise subspace. Thus, the capacity of this DOA estimation is equal to the rank of the reciprocal subspace of the selected noise subspace and is also less than the number of the antenna elements. In ESPRIT techniques, two virtual subarrays structures are proposed to obtain two signal subspaces. The eigenvectors of the relevant signal subspaces are rotated for the DOAs of the target signals. As a result, the capacity of DOA estimation using ESPRIT is bounded by the number of subarrays.

The disadvantage of the above application techniques is that the number of signal sources is less than that of antenna elements $[11,12]$. In addition, these techniques also require subspace estimation, eigen decomposition, and inversion computation of the covariance matrix, which leads to high computational complexity, and are thereby limited to the applications where fast DOA estimation is not required [13, 14]. Furthermore, in the presence of interference, these techniques need to estimate the DOAs of all the target signals and interference, which also increases computational complexity and decreases the accuracy of DOA estimation [15].

The application of adaptive beamforming in DOA estimation has become the research focus on interference existing [16]. In [16], Wang et al. developed a new structure of DOA estimation based on subarray beamforming.

\section{实}


This technique has a clear advantage on the DOA estimation when interference exists but still needs the computation of matrix inversion which is not easy to be applied to a practical system. Based on this structure, a novel Multistage Nested Wiener Filter-based (MSNWF) [17-20] DOA estimation technique (MSNWF-DOA) is proposed in this paper. This technique uses two subarray adaptive beamformers based on the MSNWF to construct the same array geometry for forming the phase shift and rejecting interference at the same time. The DOAs of the target signals are estimated from the phase shifts by using a reference signal after the rejection of interference. Therefore, the performance of DOA estimation is significantly improved. This technique can be widely used for the implementation of hardware systems such as wireless communication system, active radar, sonar, space-time adaptive process (STAP) systems [20, 21], and multiple input and multiple output (MIMO) systems [22].

The advantages of the MSNWF-DOA estimation are as follows:

1) Since the use of MSNWF in this technique realizes the subspace eigen decomposition, computation of inversion of the covariance matrix becomes unnecessary and thus reduces the complexity of computation; the MSNWF-DOA can be easily applied in hardware platform [23].

2) The capacity of DOA estimation in the proposed MSNWF-DOA technique can be far larger than the number of antenna elements.

3) In MSNWF estimation techniques, the target DOA is estimated after interference rejection [24]. In this way, the estimation resolution and accuracy of MSNWF-DOA are significantly improved.

The paper is organized as follows: In Section 2, the signal model is described using a uniform linear array system. In the Section 3, the basic structure of the MSNWF-DOA estimation system, the MSNWF-based adaptive beamforming, and the DOA computing of the proposed method are presented. Design examples and simulation results are given in Section 4 to show the performance of resolution, capacity, and the effects of snapshot length and the stages of MSNWF. Conclusions are drawn in Section 5.

\section{Signal model}

Consider a uniform linear array (ULA) system that uses $M$ elements with adjacent element spacing $d$, deployed at a base station. Assume the numbers of narrowband signals and unknown interference sources are $K$ and $P$, respectively. And these signals are received by the ULA system with different DOAs $\theta_{k}, k=1,2, \cdots, K+P$.
Using complex envelope representation, the received signals can be expressed by

$$
\mathbf{x}(t)=\sum_{k=1}^{K+P} \mathbf{a}\left(\theta_{k}\right) s_{k}(t)+\mathbf{n}(t)
$$

where $s_{k}(t)$ denotes the $k$ th signal component, $k=1,2$, $\cdots, K$ for target components, and $k=K+1, K+2, \cdots$, $K+P$ for interference components. The $\mathbf{a}\left(\theta_{k}\right)$ denotes the steering vector of the array in direction $\theta_{k}$, which is given by

$$
\mathbf{a}\left(\theta_{k}\right)=\left[1, e^{-j 2 \pi d \sin \left(\theta_{k}\right)}, \cdots, e^{-j 2 \pi d(M-1) \sin \left(\theta_{k}\right)}\right]^{\mathrm{T}}
$$

and $n(t)$ denotes a spatially stationary background noise vector with zero mean, and the cross-covariance is expressed as

$$
E\left[n\left(t_{1}\right) n^{\mathrm{H}}\left(t_{2}\right)\right]=\sigma^{2} \delta\left(t_{1}-t_{2}\right) \mathbf{I}
$$

where $\mathbf{I}$ is the identity matrix.

Suppose that the received vector $\mathbf{x}(t)$ is sampled at $n$, $n=1,2, \cdots, L$, the received signal in the matrix notation can be expressed as

$$
\mathbf{X}=\mathbf{A}(\theta) \mathbf{S}+\mathbf{N}
$$

where $\mathbf{X}$ and $\mathbf{N}$ are $M \times L$ matrices,

$$
\mathbf{X}=[\mathbf{x}(1), \mathbf{x}(2), \cdots, \mathbf{x}(L)]
$$

and

$$
\mathbf{N}=[\mathbf{n}(1), \mathbf{n}(2), \cdots, \mathbf{n}(L)]
$$

and $\mathbf{A}(\theta)$ is a $M \times K$ matrix, which is expressed as

$$
\mathbf{A}(\theta)=\left[\mathbf{a}\left(\theta_{1}\right), \mathbf{a}\left(\theta_{2}\right), \cdots, \mathbf{a}\left(\theta_{K}\right)\right]
$$

and $\mathbf{S}$ is a $K \times L$ matrix, which is expressed as

$$
\mathbf{S}=[\mathbf{s}(1), \mathbf{s}(2), \cdots, \mathbf{s}(L)]
$$

\section{MSNWF-DOA estimation}

The proposed MSNWF-DOA uses the uniform linear antenna array at the received end, and the geometry of the array is similar to that used in ESPRIT techniques. The antenna array is decomposed into equal-sized subarrays, where two subarrays are used in conjunction with two subarray MSNWF adaptive beamformers to obtain an optimal estimation of a phase-shift reference signal whose phase relative to that of the reference signal is a function of the target DOA. The target DOA is then computed from the estimated phase shift between the reference signal $\mathbf{r}_{k}$ and the phase-shifted reference signal $e^{j \phi_{k}} \mathbf{r}_{k}$. The block diagram of the MSNWF-DOA system is illustrated in Fig. 1. 


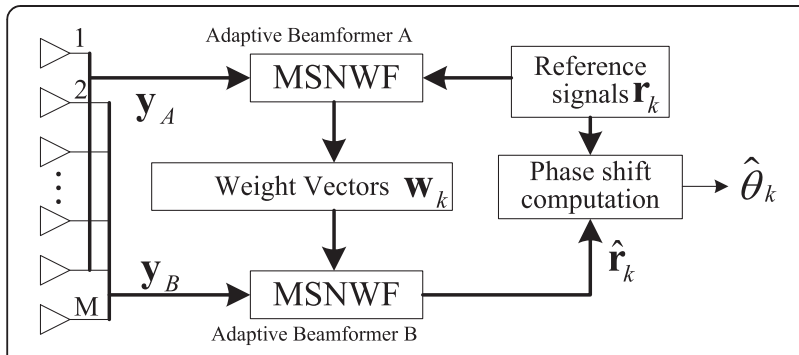

Fig. 1 Block diagram of the MSNWF-DOA system

\subsection{Subarray signal formation}

Consider that the array is composed by a ULA of $M$ element as a receiver and decomposed into two sets of $M-1$ element virtual subarrays, $A$ and $B$. The downconverted baseband signal received by the $m$ th, $m=1,2$, $\cdots, M$ element of the antenna array is expressed by

$$
x_{m}(n)=\sum_{k=1}^{K+P} e^{j(m-1) \phi_{k}} s_{k}(n)
$$

The vectors of the $A$ and $B$ are given by

$$
\mathbf{y}_{A}(n)=\left[x_{1}(n), x_{2}(n), \cdots, x_{M-1}(n)\right]^{\mathrm{T}}
$$

and

$$
\mathrm{y}_{\mathrm{B}}(\mathrm{n})=\left[\mathrm{x}_{2}(\mathrm{n}), \mathrm{x}_{3}(\mathrm{n}), \cdots \mathrm{x}_{\mathrm{M}}(\mathrm{n})\right]^{\mathrm{T}},
$$

respectively. Let

$$
\mathbf{b}\left(\theta_{k}\right)=\left[1, e^{j \phi_{k}}, \cdots, e^{j \phi_{k}(M-2)}\right]^{\mathrm{T}}
$$

Then, the subarray signals $\mathbf{y}_{A}(n)$ and $\mathbf{y}_{B}(n)$ can be written as

$$
\mathbf{y}_{A}(n)=\sum_{k=1}^{K+P} \mathbf{b}\left(\theta_{k}\right) s_{k}(n)+\mathbf{n}_{A}(n)
$$

and

$$
\mathbf{y}_{B}(n)=\sum_{k=1}^{K+P} e^{j \phi_{k}} \mathbf{b}\left(\theta_{k}\right) s_{k}(n)+\mathbf{n}_{B}(n)
$$

where vectors $\mathbf{n}_{A}(n)$ and $\mathbf{n}_{B}(n)$ are the background noise at the subarray, respectively. The phase-shift factor between the $k$ th components of signals $\mathbf{y}_{A}(n)$ and $\mathbf{y}_{B}(n)$ which from the $k$ th signal is given by

$$
e^{j \phi_{k}}=e^{-j 2 \pi d \sin \left(\theta_{k}\right) / \lambda}
$$

Sampling $\mathbf{y}_{A}(n)$ and $\mathbf{y}_{B}(n)$, we can obtain

$$
\begin{aligned}
& \mathbf{Y}_{A}=\left[\mathbf{y}_{A}(1), \mathbf{y}_{A}(2), \cdots, \mathbf{y}_{A}(L)\right] \\
& \mathbf{Y}_{B}=\left[\mathbf{y}_{B}(1), \mathbf{y}_{B}(2), \cdots, \mathbf{y}_{B}(L)\right]
\end{aligned}
$$

\subsection{Recursion algorithm of MSNWF}

In the Wiener filter, the estimation of the desired signal $d_{0}(n)$ from an observation vector $\mathbf{x}_{0}(n)$ is optimal in the minimum mean square error (MMSE) sense. The weight vector $\mathbf{w}_{\mathbf{x} 0}$ of the Wiener filter can be obtained via solving the following Wiener-Hopf equations

$$
\mathbf{R}_{\mathbf{x} 0} \mathbf{w}_{\mathbf{x} 0}=\mathbf{r}_{\mathbf{x} 0 \mathbf{d} 0}
$$

where $\mathbf{R}_{\mathbf{x} 0}$ is the covariance matrix of $\mathbf{x}_{0}(n)$, and $\mathbf{r}_{\mathbf{x} 0}$ is the cross-correlation vector between $\mathbf{x}_{0}(n)$ and $d_{0}(n)$. The covariance matrix $\mathbf{R}_{\mathbf{x} 0}$ cannot be readily estimated, if $\mathbf{x}_{0}(n)$ is of high dimension. Based on this, Goldstein and Reed proposed that if the observation $\mathbf{x}_{0}(n)$ is prefiltered by a full-rank matrix $\mathbf{T} \in \mathbb{C}^{M \times M}$, i.e., $\mathbf{z}_{1}(n)=$ $\mathbf{T x}_{0}(n)$, then the Wiener filter with the weight of $\mathbf{w}_{\mathbf{z} 1}$ which estimates $d_{0}(n)$ from $\mathbf{z}_{1}(n)$ results in the same MSE [9, 10, 16]. The assumed full-rank pre-filtering matrix can be chosen as

$$
\mathbf{T}_{1}=\left[\begin{array}{l}
\mathbf{h}_{1}^{\mathrm{H}} \\
\mathbf{B}_{1}
\end{array}\right]
$$

where $\mathrm{H}$ is the complex conjugate transpose operator. Thus, the new observation vector can be written as

$$
\mathbf{z}_{1}(n)=\left[\begin{array}{l}
\mathbf{h}_{1}^{\mathrm{H}} \mathbf{x}_{0}(n) \\
\mathbf{B}_{1} \mathbf{x}_{0}(n)
\end{array}\right]=\left[\begin{array}{c}
d_{1}(n) \\
\mathbf{x}_{1}(n)
\end{array}\right]
$$

where $\mathbf{B}_{1}$ is referred to the blocking matrix, $\mathbf{B}_{1} \mathbf{h}_{1}=0$, and $\mathbf{h}_{1}=\mathbf{r}_{\mathbf{x} 0 \mathbf{d} 0} /\left\|\mathbf{r}_{\mathbf{x} 0 \mathbf{d} 0}\right\|_{2}$.

The solution of the Wiener-Hopf equations relative to the transformed system is

$$
\mathbf{w}_{\mathbf{z} 1}=\mathbf{R}_{\mathbf{z} 1}^{-1} \mathbf{r}_{\mathbf{z} 1 \mathbf{d} 0}=\alpha_{1}\left[\begin{array}{c}
1 \\
-\mathbf{R}_{\mathbf{x} 1}^{-1} \mathbf{r}_{\mathbf{x} 1 \mathbf{d} 1}
\end{array}\right]
$$

where $\alpha_{1}=\left\|\mathbf{r}_{\mathbf{x} 0 \mathbf{d} 0}\right\|_{2} /\left(\sigma_{d 1}^{2}-\mathbf{r}_{\mathbf{x} 1 \mathbf{d} 1}^{\mathrm{H}} \mathbf{R}_{\mathbf{x} 1}^{-1} \mathbf{r}_{\mathbf{x} 1 \mathbf{d} 11}\right), \mathbf{R}_{\mathbf{z} 1}$ is the covariance matrix of the new observation vector $\mathbf{z}_{1}(n)$, and $\mathbf{r}_{\mathbf{z} 1 \mathrm{~d} 1}$ is the cross-correlation vector between the new desired signal $d_{1}(n)$ and the new observation vector $\mathbf{z}_{1}(n)$. And $\mathbf{R}_{\mathbf{x} 1}=\mathbf{B}_{1} \mathbf{R}_{\mathbf{x} 0} \mathbf{B}_{1}^{\mathrm{H}}$ is the covariance matrix of the new observation vector $\mathbf{x}_{1}(n), \sigma_{d 1}^{2}=\mathbf{h}_{1}^{\mathrm{H}} \mathbf{R}_{\mathbf{x} 0} \mathbf{h}_{1}$ is the variance of the new desired signal $d_{1}(n)$, and $\mathbf{r}_{\mathbf{x} 1 \mathbf{d} 1}=\mathbf{B}_{1} \mathbf{R}_{\mathbf{x} 0} \mathbf{h}_{1}$ is the cross-correlation vector between $\mathbf{x}_{1}(n)$ and $d_{1}(n)$.

The transform process produces a new vector Wiener filter, which estimates the signal $d_{1}(n)$ from the observation vector $\mathbf{x}_{1}(n)$, and a scalar Wiener filter is followed. Repeating this process, a nested Wiener filter structure can be obtained, which is defined as the original Multistage Nested Wiener Filter [16-19]. In addition, the group of orthogonal weight vectors extracted by earlier several forward recursions spans the signal subspace, 
which ensures that the MSNWF algorithm is completed for the estimation of the direction of arrival and the reduced-rank adaptive filtering. In order to avoid the formation of blocking matrices required in the original algorithm, Zoltowski et al. proposed a data level recursive MSNWF algorithm [23, 24] as shown in Fig. 2, which effectively reduces the computational complexity.

In Fig. $2, \mathbf{t}_{i}, i=1,2, \cdots, D$ is a match filter, $D$ is the recursive stage, $\varepsilon_{i}, i=1,2, \cdots, D$ is MSE at the $i$ th stage, and $\omega_{i}, i=1,2, \cdots, D$ is the coefficient of Wiener filter calculated at the $i$ th stage.

The flow diagram of the data level recursion MSNWFDOA estimation algorithm is given in Table 1.

\subsection{MSNWF-DOA estimation system}

In the MSNWF-DOA system, the optimal estimation of the phase-shifted reference signal $e^{j \phi_{k}} \mathbf{r}_{k}$ in the minimum mean square error sense can be obtained at the output of the adaptive beamformer $B$, where the adaptive beamforming weights obtained from the adaptive beamformer $A$ with the MSNWF structure were used.

In the adaptive beamformer $B$, consider the case where the phase-shifted reference signal $e^{j \phi_{k}} \mathbf{r}_{k}$ is the desired signal, and the output of the adaptive beamformer $B$ can be used to estimate the desired signal. Since the phaseshifted $e^{j \phi_{k}}$ is unknown, both the phase-shifted reference signal and the weight vector of the adaptive beamformer $B$ are not available. However, the weight vector of the adaptive beamformer $B$ can be obtained from the optimal weights of the adaptive beamformer $A$, which is shown as follows:

In the adaptive beamformer $A$, the desired signal and observation vector can be given by

$$
\left\{\begin{array}{c}
d_{A 0}(n)=r_{k} \\
\mathbf{x}_{A 0}(n)=\mathbf{y}_{A}(n)
\end{array}\right.
$$

The optimal weight vector of adaptive beamformer $A$ can be readily obtained according to Table 1 as shown in Table 2.

In the adaptive beamformer $B$, the phase-shifted desired signal and observation vector can be given by

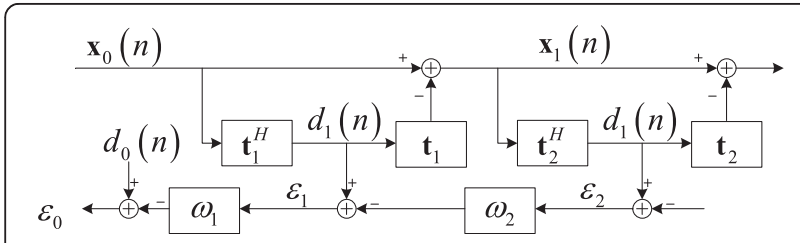

Fig. 2 The structure of data level recursive MSNWF
Table 1 Date level recursive MSNWF

Forward recursion for $i=1,2, \cdots, D$
$\mathbf{t}_{i}=\sum_{n=1}^{L-1} d_{i-1}^{*}(n) \mathbf{x}_{i-1}(n)$
$t_{\mathbf{i}}=t_{\mathbf{i}} /\left\|t_{\mathbf{i}}\right\|_{\mathbf{2}}$
$d_{i}(n)=\mathbf{t}_{i}^{H} \mathbf{x}_{i-1}(n), n=1, \cdots, L-1$
$\mathbf{x}_{i}(n)=\mathbf{x}_{i-1}(n)-d_{i}(n) \mathbf{t}_{i}, n=1, \cdots, L-1$
$\varepsilon_{D}(n)=d_{D}(n), n=1, \cdots, L-1$

Backward recursion for $i=D-1, \cdots, 2,1$

$\omega_{i+1}=\sum_{n=1}^{L-1} d_{i}(n) \varepsilon_{i+11}^{*}[n] / \sum_{n=1}^{L-1}\left|\varepsilon_{i+1}(n)\right|^{2}$

$\varepsilon_{j}(n)=d_{i}(n)-\omega_{i}+{ }_{1} \varepsilon_{i+1}(n), n=1, \cdots, L-1$

Calculate the Wiener filter coefficient

$\mathbf{w}^{(D)}=\sum_{i=1}^{D}(-1)^{i+1}\left\{\prod_{i=1}^{i}\left(-\omega_{l}\right)\right\} \times \mathbf{t}_{i}$

$$
d_{B 0}(n)=e^{j \phi_{k}} \mathbf{r}_{k}
$$

And the optimal weight vector of the adaptive beamformer $B$ can be obtained according to Table 1 as shown in Table 3.

Substitute (13), (14), and (16) into Table 3, we have

$$
\mathbf{t}_{A(1)}=\mathbf{t}_{B(1)}
$$

and

$$
\mathbf{w}_{k}^{A(D)}=\mathbf{w}_{k}^{B(D)}
$$

Since $\mathbf{w}_{k}^{A(D)}=\mathbf{w}_{k}^{B(D)}$, the weight vector $\mathbf{w}_{k}^{B(D)}$ can be obtained by calculating the optimal weight of the adaptive beamformer $A$.

\section{Computation of DOA}

The adaptive beamformer $B$ based on the structure of MSNWF can be simplified to a single stage Wiener filter in virtue of obtaining its weight from the adaptive

Table 2 The flow diagram of computation of weight vector in adaptive beamformer $A$

Initialization

$\mathbf{t}_{A(1)}=r_{k}^{*} \mathbf{y}_{A}(n) /\left\|r_{k}^{*} \mathbf{y}_{A}(n)\right\|_{2}, n=1, \cdots, L-1$

Forward recursion for $i=2,3, \cdots, D$

$\mathbf{t}_{A(i)}=\sum_{n=1}^{L-1} d_{A(i-1)}^{*} \mathbf{x}_{A(i-1)}(n)$

$\mathbf{t}_{A(i)}=\mathbf{t}_{A(i)}\left\|\mathbf{t}_{A(i)}\right\|_{2}$

$d_{A(i)}(n)=\mathbf{t}_{A(i)}^{H} \mathbf{x}_{A(i-1)}(n), n=1, \cdots, L-1$

$\begin{aligned} \mathbf{x}_{A(i)}(n)= & \mathbf{x}_{A(i-1)}(n)-d_{A(i)}(n) \mathbf{t}_{A(i)}, \\ & n=1, \cdots, L-1\end{aligned}$

$\varepsilon_{A(D)}(n)=d_{A(D)}(n), n=1, \cdots, L-1$

Backward recursion for $i=D-1, \cdots, 2$

$\omega_{A(i+1)}=\sum_{\mathcal{A}^{\prime}}^{L-1} d_{A(i)}(n) \varepsilon_{(i+1)}^{*}(n) / \sum_{n=1}^{L-1}\left|\varepsilon_{A(i+1)}(n)\right|^{2}$
$\varepsilon_{A(i)}(n)=d_{A(i)}(n)-\omega_{A(i+1)} \varepsilon_{A(i+1)}(n)$,
$n=1, \cdots, L-1$

Calculate the Wiener filter coefficient

$\underline{\mathbf{w}_{k}^{A(D)}=\sum_{i=1}^{D}(-1)^{i+1}\left\{\prod_{i=1}^{i}\left(-\omega_{A(l)}\right)\right\} \times \mathbf{t}_{A(i)}}$ 
Table 3 The flow diagram of computation of weight vector in adaptive beamformer $B$

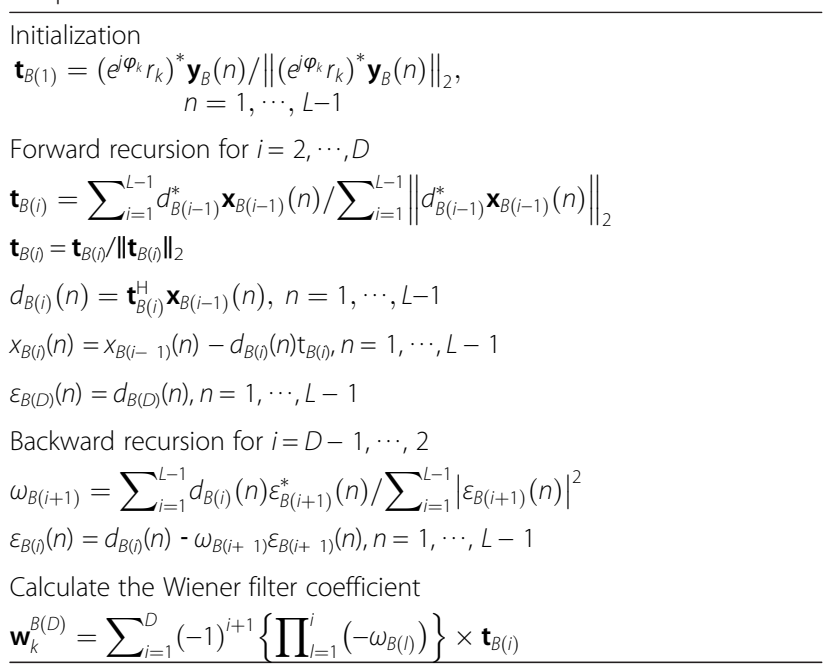

beamformer $A$. Let $\hat{\mathbf{r}}_{k}(n)=\left(\mathbf{w}_{k}^{B(D)}\right)^{\mathrm{H}} \mathbf{y}_{B}$ denote the output signal of beamformer $B$. Let

$$
\widehat{\mathbf{r}}_{k}=\left[\widehat{\mathbf{r}}_{k}(1), \overline{\mathbf{r}}_{k}(2), \cdots, \overline{\mathbf{r}}_{k}(L)\right]^{\mathrm{T}}
$$

Thus, $\hat{\mathbf{r}}_{k}$ is an optimal estimation of the phase-shifted reference signal $e^{j \phi_{k}} \mathbf{r}_{k}$ in the MMSE sense, which can be written as

$$
\hat{\mathbf{r}}_{k}=e^{j \phi_{k}} \mathbf{r}_{k}+\mathbf{N}
$$

Let $\bar{\phi}_{k}$ denote an estimation of $\phi_{k}$, which can be computed by using the least square method such that the square error between the two signal vectors $\hat{\mathbf{r}}_{k}$ and $\mathbf{r}_{k}$ is minimized, i.e.,

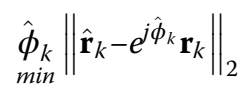

In [10], Zhang et al. give the solution of the optimization $\bar{\phi}_{k}$, that is

$$
\hat{\phi}_{k}=\arg \left(\hat{\mathbf{r}}_{k} \mathbf{r}_{k}^{\mathrm{H}}\right)
$$

According to (15), an estimation of the target DOA can then be obtained as

$$
\hat{\theta}_{k}=\arcsin \left(-\lambda \hat{\phi}_{k} / 2 \pi d\right)
$$

\section{Simulations}

In this section, the performance of the proposed method, including the resolution, capacity, and accuracy of the MSNWF-DOA techniques will be evaluated through simulations. In simulations 1 and 2, the resolution and the capacity of the DOA estimation using the MSNWF-DOA techniques will be illustrated and compared with other techniques, such as MUSIC and ESPRIT. In simulations 3 and 4, the effects of the snapshot length and the stage of MSNWF on the estimation accuracy will be investigated, respectively.

\subsection{Resolution of DOA estimation}

A ULA of ten elements, with a spacing of $d=\lambda / 2$ deployed at the receiver was employed in the simulations, to deal with a case where the DOAs of three signals and two interference are closely distributed. Further assume that the DOAs of the target incoherent signal components are at $-2^{\circ}, 0^{\circ}$, and $2^{\circ}$. The DOAs of the interference components are at $-4^{\circ}$ and $4^{\circ}$. The information bit-to-background noise power spectral density ratio of the received signal is set to $10 \mathrm{~dB}$. The snapshot length is fixed at 100, and the stage of MSNWF is set to 5. One thousand simulation runs were performed.

The histograms of the resolution of DOA estimation obtained for these three techniques are shown in Fig. 3a-d. The histogram depicts the number of occurrences estimated DOA as a function of DOA degrees. In Fig. 3a, the histogram of MUSIC technique shows two peak values which deviate from the DOAs of the target signals. In Fig. 3b, although the histogram of ESPRIT technique shows three peak values, the peak values deviate from the DOAs of the target signals. It is seen that the MUSIC or ESPRIT technique cannot offer the desired results when the DOAs of target signals are very close. Correspondingly, in Fig. 3c, d, the histogram shows three peak values, indicating that using the subarray beamforming-based DOA (SBDOA) and the proposed MSNWF-DOA, all three DOAs are successfully estimated. However, the SBDOA requires $O\left(\mathrm{M}^{3}\right)$ operations, and the MSNWF-DOA merely demands $O\left(2 \mathrm{M}^{2}\right.$ $+6 \mathrm{M})$ operations.

\subsection{Capacity of DOA estimation}

This simulation deals with a case where the number of signals is larger than that of antenna elements. The simulation conditions are kept the same as those in simulation 1 except for the number of signal sources considered. The DOAs of nine target signal components are set from $-40^{\circ}$ to $40^{\circ}$ with interval $10^{\circ}$, and the DOAs of six interference components are set from $-25^{\circ}$ to $25^{\circ}$ with interval $10^{\circ}$.

Histograms of the obtained estimated DOAs are shown in Fig. 4a-d. In Fig. 4a, b, the histograms show the deviated peak values and demonstrate that these two techniques cannot provide acceptable DOA estimation, when the number of antenna elements is less than the total number of target signals and interference. In contrast, in Fig. 4c, d, the histograms show that all nine target DOAs are successfully estimated when using the SBDOA and MSNWF-DOA techniques. As can be seen, the probability of the success of DOA estimation in 


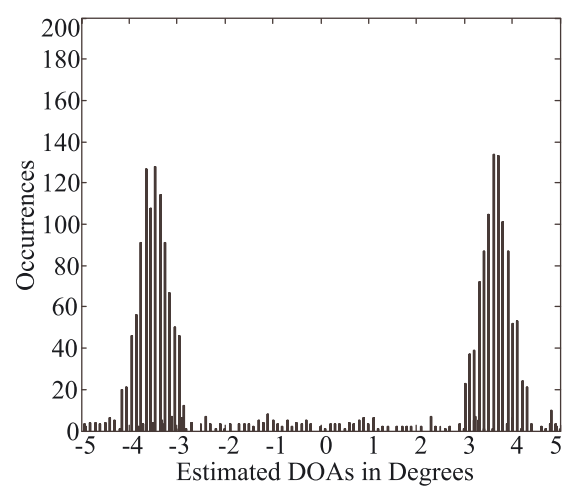

(a) Resolution of MUSIC estimation

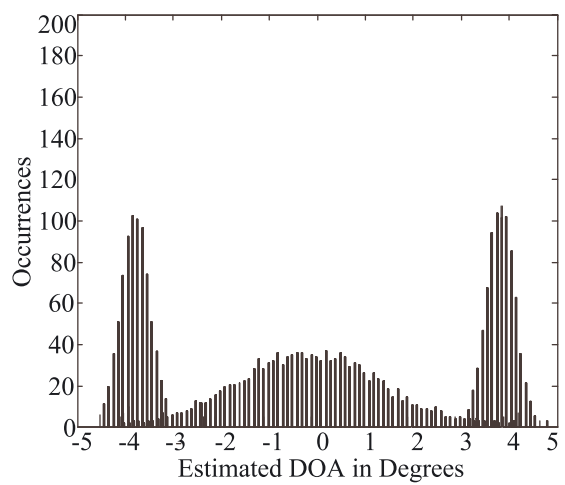

(b) Resolution of ESPRIT estimation

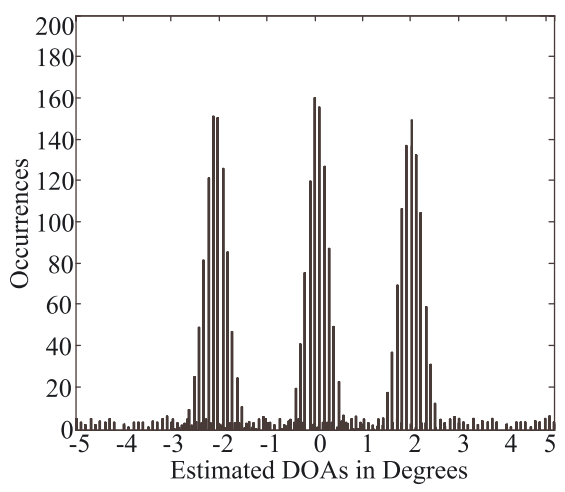

(c) Resolution of SBDOA estimation

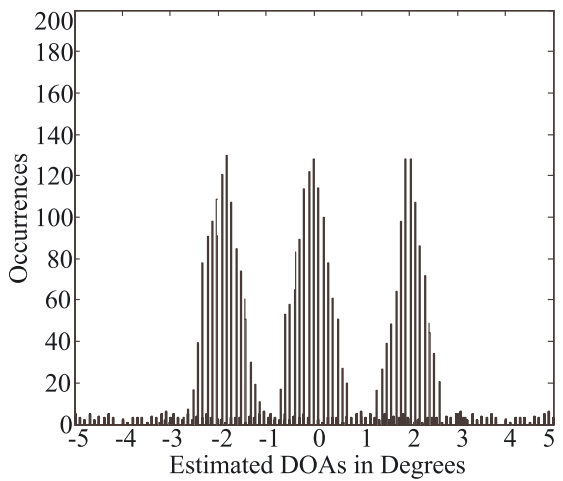

(d) Resolution of MSNWF-DOA estimation

Fig. 3 Comparison of the resolution of DOA estimation for signal sources that are closely distributed. a Resolution of MUSIC estimation. $\mathbf{b}$ Resolution of ESPRIT estimation. c Resolution of SBDOA estimation. $\mathbf{d}$ Resolution of MSNWF-DOA estimation

MSNWF-DOA technique is close to that in the SBDOA technique.

\subsection{Effects of snapshot length on estimation accuracy}

In the simulation of snapshot length effect, the snapshot length for adaptive beamforming and DOA computation are set to different values, 20,50,100, 200, and 500, and the stage of MSNWF is set to five. The DOA of the target signal is fixed at $0^{\circ}$, and the DOAs of the interference are set from $-90^{\circ}$ to $90^{\circ}$ with interval $10^{\circ}$ except $0^{\circ}$. The root mean square error (RSME) of the estimated target DOA averaged over 1000 simulation runs versus the signal-to-noise ratio (SNR) of the target DOAs and the snapshot length are illustrated in Fig. 5.

The proposed MSNWF-DOA technique leads to a RSME of less than $5^{\circ}$, as can be seen, when using a small snapshot length such as 50. The RSME obviously decreases as the snapshot length increases. This demonstrates the fast DOA tracking can be implemented by using the proposed MSNWF-DOA technique.

\subsection{Effects of the stage of MSNWF on DOA estimation accuracy}

In the simulation of the MSNWF stage effect, the recursion stages of MSNWF for adaptive beamforming are set to different values, 3, 5, and 9. The snapshot length is set to 200. And other simulation conditions are kept the same as those in the simulation for the snapshot length effect. The RSME of the estimated target DOA averaged over 10,000 simulation runs versus the SNR of the target DOAs, and the stage of MSNWF are demonstrated in Fig. 6. The SBDOA can be considered as the full-rank of MSNWF-DOA. The RSME decreases as the MSNWF stage increases. This figure shows that the RSME is less than $2.1^{\circ}$, when using stage 5 as the MSNWF stage, and the RSME of MSNWF-DOA is better than the RSME of SBDOA, when using stage 9 as the MSNWF stage.

\section{Conclusions}

A novel DOA estimation method based on data level recursive MSNWF has been proposed in this paper. In this technique, two subarray adaptive beamformers based on the MSNWF are used to form the phase shift and reject interference at the same time. The DOAs of target signals are estimated from the phase shift by using a reference signal after interference rejection. Therefore, the performance of DOA estimation such as resolution, capacity, and accuracy are significantly improved. And the complexity of computation is also significantly reduced by avoiding the calculation of the covariance matrix 


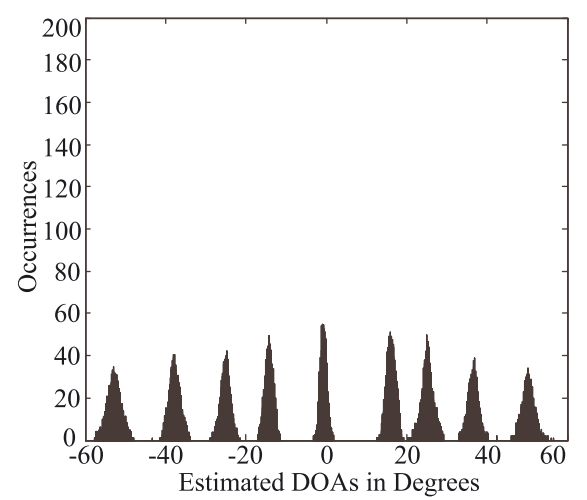

(a) Capacity of MUSIC estimation

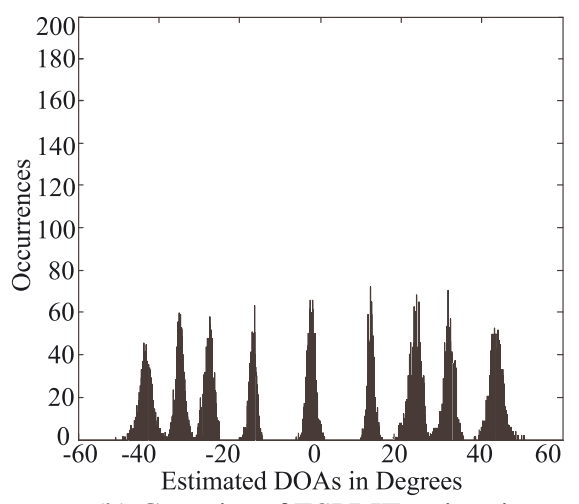

(b) Capacity of ESPRIT estimation

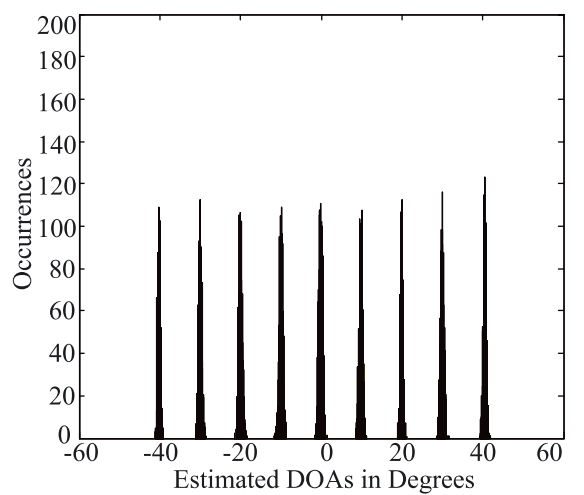

(c) Capacity of SBDOA estimation

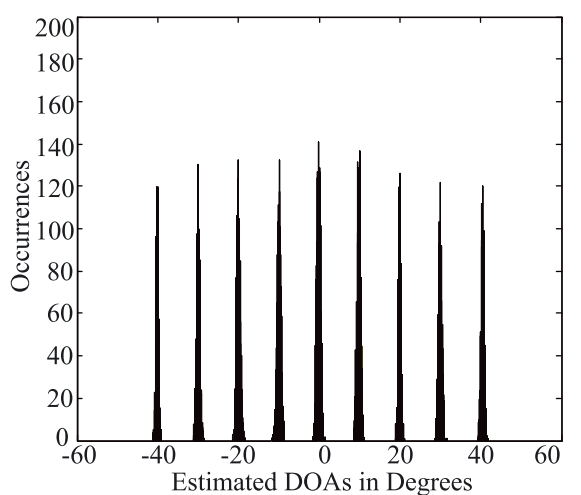

(d) Capacity of MSNWF-DOA estimation

Fig. 4 Comparison of the capacity of DOA estimation when the number of signal and interference sources exceeds the number of antenna elements. a Capacity of MUSIC estimation. b Capacity of ESPRIT estimation. c Capacity of SBDOA estimation. $\mathbf{d}$ Capacity of MSNWF-DOA estimation

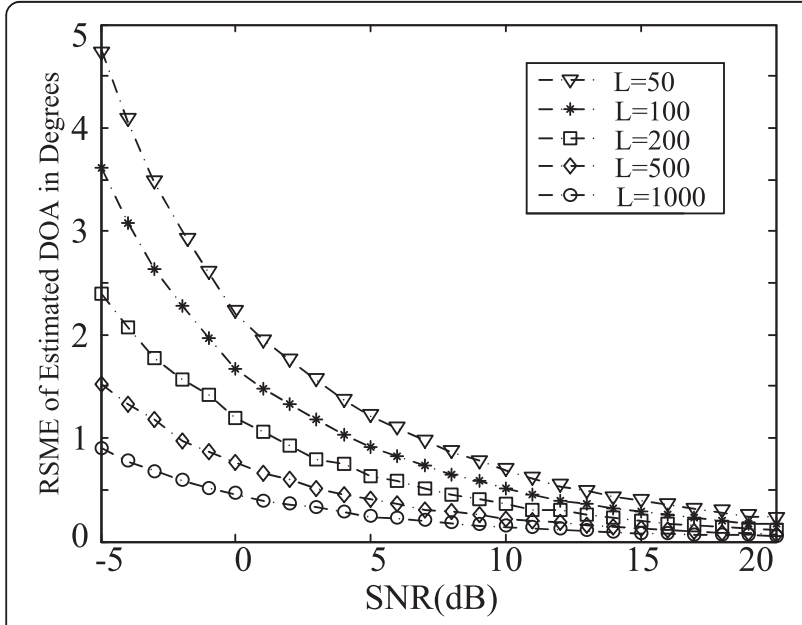

Fig. 5 RSME of the estimated DOA for different snapshot length $L$ and the SNR of signal

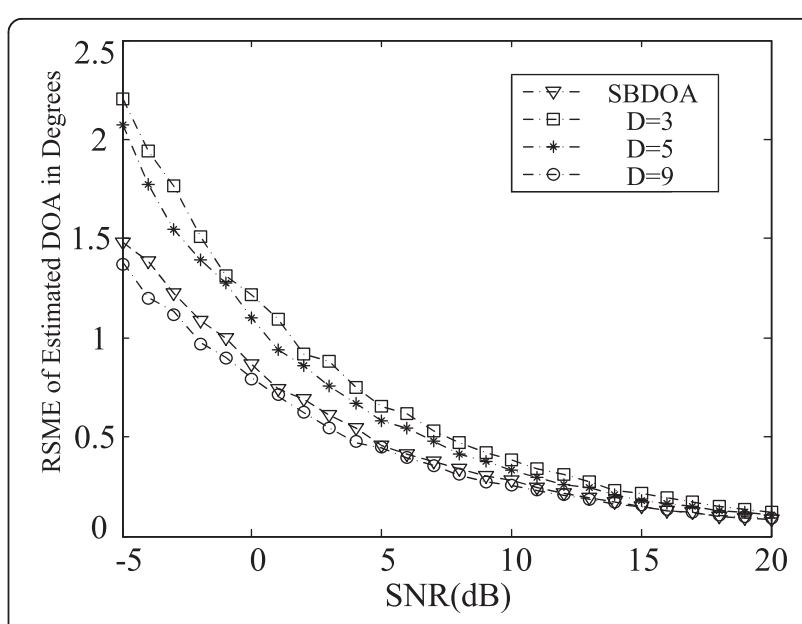

Fig. 6 RSME of the estimated DOA for different recursion MSNWF stage $D$ and the SNR of signal 
inversion when getting the optimal weight vector of the beamformer. This technique can be widely used for the implementation of hardware systems such as wireless communication system, active radar, sonar, STAP systems, and MIMO systems. Numerical simulations demonstrating the effectiveness and advantage of this technique are presented.

\section{Competing interests}

The authors declare that they have no competing interests.

Received: 18 July 2014 Accepted: 11 May 2015

Published online: 03 June 2015

\section{References}

1. JC Liberti, TS Rappaport, Smart Antennas for Wireless Communication. IS-95 and Third Generation CDMA Applications (Prentice Hall, Englewood Cliffs, NJ, 1999)

2. LC Godara, Application of antenna arrays to mobile communications. II: beam-forming and direction-of-arrival considerations. Proc. IEEE 85(8), 1195-1245 (1997)

3. A Klouche-Djedid, M Fujita, Adaptive array sensor processing applications for mobile telephone communications. IEEE Trans Vehicular Technol. 45(3), 405-416 (1996)

4. MS Bartlett, Periodogram analysis and continuous spectra. Bimetrica 37, $1-16(1950)$

5. J Capon, High-resolution frequency-wave-number spectrum analysis. Proc. IEEE 57(8), 1408-1418 (1969)

6. $\mathrm{HH}$ Chen, SC Chan, ZG Zhang, KL Ho, Adaptive beamforming and recursive DOA estimation using frequency-invariant uniform concentric spherical arrays. IEEE Trans Circuits Syst-I Reg Papers. 55(10), 3077-3089 (2008)

7. RO Schmidt, Multiple emitter location and signal parameter estimation. IEEE Trans Antennas Propagation. 34(3), 276-280 (1986)

8. R Roy, T Kailath, ESPRIT_estimation of signal parameters rotational invariance techniques. IEEE Trans Acoustics Speech Signal Process. 37(7), 984-995 (1989)

9. P Strobach, Fast recursive subspace adaptive ESPRIT algorithms. IEEE Trans. Signal Process. 46(9), 2413-2429 (1998)

10. XF Zhang, X Gao, DZ Xu, Multi-invariance ESPRIT-based blind DOA estimation for MC-CDMA with an antenna array. IEEE Trans. Vehicular Technol. 58(8), 4686-4690 (2009)

11. W Myrick, MD Zoltowski, Low-sample performance of reduced-rank power minimization based jammer suppression for GPS (IEEE Sixth International Symposium on Spread Spectrum Techniques \& Applications (ISSSTA 2000), Parsippany, NJ, USA, 2000), pp. 93-97

12. ML Honig, W Xiao, Performance of reduced-rank linear interference suppression. IEEE Trans. Inform. Theory 47, 1928-1946 (2001)

13. R Singh, LB Milstein, Interference suppression for DS/CDMA. IEEE Trans. Commun. 47, 446-453 (1999)

14. W Xiao, ML Honig, Convergence Analysis of Adaptive Full-Rank and Multistage Reduced-Rank Interference Suppression (Information Sciences and Systems Conference, Princeton, NJ, 2000)

15. WL Myrick, MD Zoltowski, JS Goldstein, Adaptive anti-jam reduced-rank space-time pre-processor algorithm for GPS (Institute of Navigation (ION) conference, Salt Lake City, Utah, USA, 2000), pp. 321-336

16. NY Wang, P Agathoklios, A Antoniou, A new DOA estimation technique based on subarray beamform. IEEE Trans. Signal Process. 54(9), 3279-3290 (2006)

17. JS Goldstein, IS Reed, A new method of Wiener filtering and its application to interference mitigation for communications. MILCOM Proc 3, 1087-1091 (1997)

18. JS Goldstein, IS Reed, Reduced rank adaptive filtering. IEEE Trans. Signal Process. 45(2), 492-496 (1997)

19. JS Goldstein, IS Reed, LL Scharf, A multistage representation of the Wiener filter based on orthogonal projections. IEEE Trans. Inf. Theory 44(7), 2943-2959 (1998)

20. JS Goldstein, IS Reed, Multidimensional Wiener Filtering Using a Nested Chain of Orthogonal Scalar Wiener Filters (USC Tech. Rep. CSI-96-12-04, University of Southern California, Los Angeles, CA, 1996)
21. ML Honig, JS Goldstein, Adaptive reduced-rank interference suppression based on the Multistage Wiener Filter. IEEE Trans. Commun. 50(6), 986-994 (2002)

22. HH Chen, ZG Pan, L Tian, JL Shi, GH Yang, M Suzuki, A Novel, AWSF algorithm for DOA estimation in virtual MIMO systems. IEEE J Selected Areas Commun. 31(10), 1994-2003 (2013)

23. MD Zoltowski, E Santos, Advance in reduced-rank adaptive beamforming, vol. 5540 (SPIE 2004 Defense and Security Symposium, Orlando, FL, USA, 2004)

24. MD Zoltowski, M Joham, S Chowdhury, Recent advances in reduced-rank adaptive filtering with application to high-speed wireless communications. Proc. SPIE 2001, 482-485 (2001)

\section{Submit your manuscript to a SpringerOpen ${ }^{\odot}$ journal and benefit from:}

- Convenient online submission

- Rigorous peer review

- Immediate publication on acceptance

- Open access: articles freely available online

- High visibility within the field

- Retaining the copyright to your article

Submit your next manuscript at $>$ springeropen.com 